\title{
Effects of maternal age on larval competitiveness in house flies
}

\author{
G. S. MCINTYRE* \& R. H. GOODING \\ Department of Biological Sciences, University of Alberta, Edmonton, Canada AB T6G 2E9
}

\begin{abstract}
At advanced ages, many insects lay smaller eggs with reduced viability, but adults produced by different maternal age classes are usually indistinguishable. In most species it is not known if there are any significant differences between hatchlings from smaller, later eggs (i.e. those produced by old females) and those from larger, earlier eggs (i.e. those produced by young females). For many insects, the best way to determine if such differences exist is to rear larvae from different maternal age classes together and compare their success. We tested the effects of maternal age on the competitive ability of house fly larvae, using a modified replacement (substitution) design with pairwise comparisons of two maternal age classes from three electrophoretically marked lines. For each comparison, known numbers of larvae were reared together at five ratios, including pure cultures, at densities high enough to ensure severe competition. We measured the effects of maternal age on hatchling to adult survival, development time, and adult size. In general, older females produced larvae that had higher viability and attained larger sizes, but developed more slowly. Maternal age effects were line-specific, suggesting that they are determined genetically, and there were significant interactions of maternal age effects between pairwise line comparisons. Maternal age effects on performance in pure culture were not predictive of performance in mixed cultures. Competitor identity significantly affected the success of each line and maternal age class, suggesting that use of tester strains to determine relative competitiveness of lines, or maternal age classes, is not generally valid. The results are discussed with respect to the possible adaptive nature of maternal age effects in this species.
\end{abstract}

Keywords: ageing, larval competition, maternal effects, Musca domestica, tester strains.

\section{Introduction}

Offspring of old female insects (hereafter referred to as late offspring) are usually thought of as inferior performers, relative to the offspring of young females (hereafter referred to as early offspring). Late offspring are often smaller as hatchlings, arising from smaller eggs with lower average viability, than their earlier siblings do. They often have decreased growth rates, and larval viability (for reviews see Mousseau \& Dingle, 1991a,b; Mousseau \& Fox, 1998; McIntyre, 2000). Maternal age effects generally become attenuated as the offspring develop but in some cases they extend to later larval and even adult performance (e.g. adult size of offspring: Rossiter, 1991; McIntyre \& Gooding, 1998). But these later differences are usually measured as performance or fitness components of isolated groups or individuals. There have been few studies of maternal age effects in which offspring of young and old females interact. Therefore, the intraspecific competitive fitness of off-

*Correspondence. E-mail: gmcintyr@ualberta.ca spring of different maternal ages is essentially unknown in insects, in spite of the considerable body of research apparently addressing it.

Most maternal age effects are considered nonadaptive because they are thought to result from physiological constraints and deterioration (e.g. inability to adequately provision eggs at advanced ages: Murphy et al., 1983). However, many maternal effects are adaptive, patterned responses of females to predictive cues resulting in improved offspring success (Mousseau \& Fox, 1998). For example, the maternal age-related increase in the incidence of diapause in many seasonal populations corresponds well with the increasing need for offspring to enter diapause as the season advances (Mousseau \& Dingle, 1991a,b; Denlinger, 1998). Furthermore, in species that experience consistent environmental changes during the life of individuals, maternal age effects that improve offspring performance may exist. If, as a result of seasonal changes or resource depletion, density consistently becomes challenging to their offspring as females age, maternal effects could evolve to avoid or otherwise cope with higher larval density. An initial step 
in demonstrating that a maternal age effect is an adaptive response to increasing density is to show that old mothers produce larvae that are more successful in competition in or avoidance of high density.

A common approach used to study maternal age effects is to raise isolated groups of offspring from mothers of various ages and compare performance among these groups. This approach can not reveal maternal age effects on larval competition, which is an important fitness component in species that have significant larval interactions. As pointed out by Lewontin (1955) 'The supposition that one can predict the relative viabilities of genotypes from their absolute viabilities in pure culture is really a supposition that there is no interaction between these genotypes in exploiting the resources.' The critical experiment is to raise offspring from different aged females in competition with each other, as we report in this paper.

A common method for comparing competitive ability of two phenotypically indistinguishable strains is to compare both strains to a third, phenotypically distinct, 'tester' strain (e.g. Santos et al., 1992). However, to extend the logic of Lewontin (1955), this assumes that the different genotypes interact with each other in a common fashion. This assumption is unwarranted, unless there is a single major determinant of competitive success.

Studies on the bionomics of house fly larvae suggest that there are two particularly important phases in their development: initial medium conditioning by hatchlings and growth of the third (final) instar. Survival and adult size of house flies are determined by the performance of larvae during both of these phases. House fly larvae are bacterial feeders that actively condition their medium through the release of waste ammonium that stimulates yeast and bacterial growth (Bryant, 1969; Schmidtmann \& Martin, 1992). Insufficient early larval density may result in poor medium conditioning and death of larvae through starvation or fungal takeover of the medium (Bryant \& Sokal, 1967). However, with very high initial density, high early mortality can lead to increased total survival and larger flies through reduced density during the growth phase of the final larval instar (Bryant \& Sokal, 1968; Kence \& Jdeidi, 1997). Flies developing at elevated larval density are smaller, take longer to develop, and have lower survival (Sullivan \& Sokal, 1963; Bryant \& Sokal, 1967). Most of the negative effects of high density occur during the final larval instar, probably as a result of food depletion (Sullivan \& Sokal, 1963).

The outcome of larval competition in house flies can not be predicted reliably from performance in pure culture and it depends upon density, the identity of the competing strains, and the ratio of the strains (Sokal \&
Sullivan, 1963; Bhalla \& Sokal, 1964; Sullivan \& Sokal, 1965). In the current study we confirm these results and extend them to the impact of maternal age on competitive ability. We tested genetically marked strains against each other to address a single main question: Are there maternal age effects on competitive ability in house flies? This approach allowed us to address the following two corollaries: Do maternal age effects vary between strains? Are maternal age effects competitor-dependent? The results are discussed with respect to the possible adaptive nature of maternal age effects in house flies and the use of tester strains to determine relative competitive ability.

\section{Materials and methods}

\section{The flies}

The history and maintenance of our house fly colony was reported in McIntyre \& Gooding (1995, 2000). Three electrophoretically marked lines were selected from this colony. We used electrophoretic markers because they are more likely to be neutral than are morphological markers, some of which affect development and competition in house flies (Sokal \& Sullivan, 1963; Bhalla \& Sokal, 1964; Sullivan \& Sokal, 1965). The selected lines differed at either the glutathione reductase locus $(G r)$, the octanol dehydrogenase locus $(O d h)$, or at both loci. In this, and other papers describing work on these $M$. domestica colonies, the genetically marked lines are designated $\mathrm{C}$ (homozygous $G r^{s}, O d h^{f}$ ), D (homozygous $G r^{s}, O d h^{s}$ ) and G (homozygous $\left.G r^{f}, O d h^{f}\right)$. Polyacrylamide gel electrophoresis was used during selection and later to determine the origin of flies from the competition experiments. We used $7 \%$ gels at $\mathrm{pH} 8.9$. Each gel was stained in $25 \mathrm{~mL}$ of $50 \mathrm{~mm}$ TRIS pH 7.2 containing $9 \mathrm{mg}$ NADP, $9 \mathrm{mg}$ nitro blue tetrazolium (NBT), $3 \mathrm{mg}$ phenazine methosulphate (PMS), plus substrate; for GSH this was $3 \mathrm{mg}$ reduced glutathione and for ODH 8 drops of octanol. Under these conditions the positions of the bands, relative to the bromophenol blue front $\left(\mathrm{R}_{\mathrm{f}}\right)$ values were 0.70 for $G r^{s}, 0.74$ for $G r^{f}, 0.35$ for $O d h^{s}$ and 0.39 for $O d h^{f}$. At $O d h$ and $G r$ there were actually two very similar fast alleles. Because we were interested only in using these as markers we did not distinguish between the fast alleles in these experiments.

\section{Competition experiments: design and setup}

In order to obtain eggs and larvae for each experiment, populations of 'young' and 'old' flies were established so the youngest and oldest flies within each age group differed in age by a maximum of $48 \mathrm{~h}$ and the mean 
difference between flies from different age groups was 14 days. The left wings of a sample of 16 surplus females from each group were slide-mounted and measured as described below. Eggs were collected twice weekly, beginning at age seven days, from each group of mothers. The eggs used for the competition experiments were taken from $24 \pm 1$-d-old 'old' mothers (the sixth egg collection) and $10 \pm 1$-d-old 'young' mothers (the second egg collection). We chose these ages because 10d-old females produce the largest eggs with the highest viabilities, and 24-d-old females produce significantly smaller eggs with lower viabilities but females are not postreproductive and have not begun to suffer significant mortality (McIntyre \& Gooding, 2000).

The relative larval competitiveness of young and old house flies were examined using a modified replacement (substitution) series experiment (Mather \& Caligari, 1981; Novak et al., 1993) (Fig. 1), i.e. the groups of interest were reared with each other at several ratios with the same overall initial density. In order to investigate maternal age effects, our design was more complex because (i) we cannot simultaneously produce offspring from the same mother(s) at two different ages

\section{Experimental Design}

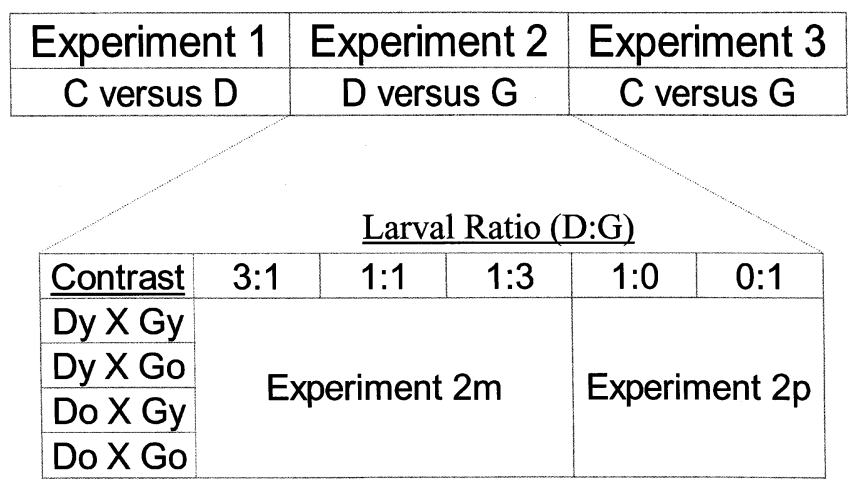

Fig. 1 Design of competition experiments: Separate experiments were conducted for each combination of the three marked lines. In each experiment we reared larvae from young and old females of each genotype separately with larvae from each maternal age class in the other line. Experiment 2 consisted of the following combinations: young D (Dy) with young G (Gy), Dy with old G (Go), old D (Do) with Gy, and Do with Go. Within each of these combinations we tested five larval seeding ratios $(1: 0,3: 1,1: 1,1: 3$, and $0: 1)$. Each experiment is divisible into two parts, tests of performance in pure cultures (seeding ratios 1:0 and 0:1; designated as expts $1 \mathrm{p}, 2 \mathrm{p}$, and $3 \mathrm{p}$ ) and tests of performance in mixed culture (seeding ratios 3:1, 1:1 and 1:3; designated as expts $1 \mathrm{~m}, 2 \mathrm{~m}$, and $3 \mathrm{~m})$. In each treatment three replicate cups were seeded with a total of 60 larvae in three grams of larval medium. and (ii) we cannot distinguish offspring from the same kind of mother. For each pairwise combination of marked lines we reared larvae from young and old females of each line in all possible combinations at five seeding ratios. Because of the fundamental difference between intra-and intergroup competition each experiment contains a logical subdivision into mixed cups (i.e. cups with larvae from two colonies; Exp. 1m, 2m, and $3 \mathrm{~m}$ ) and pure cups (i.e. cups with larvae from only one colony; Exp. 1p, 2p, and 3p). Throughout this paper we designate the genetically marked lines of $M$. domestica as 'C', 'D' and 'G', and the offspring of 10-d-old females as 'young' (abbreviated ' $y$ ') and those of 24-d-old females as 'old' (abbreviated 'o'). We also use these designations in combination; for example 'Dy' identifies offspring from young females of line $D$ and 'Go' identifies offspring of old females from line G (Fig. 1).

Eggs were collected, on cotton soaked in evaporated milk from 09.00 to 16.00 hours, at which time they were separated from the adult flies. A sample of eggs was frozen at $-20^{\circ} \mathrm{C}$ in $0.85 \%$ saline for later measurement (eggs can be frozen for up to 1 years this way without significant alteration of size or shape; McIntyre 2000). A second sample of 50 eggs was incubated for $24 \mathrm{~h}$ at $25^{\circ} \mathrm{C}$. Hatch rate was recorded for these eggs. The remainder of the eggs were incubated, on the milksoaked cotton, until 09.00 hours the following morning to allow hatching. Three replicate cups for each combination of line, age, and ratio were seeded with a total of 60 vigorous larvae in $3 \mathrm{~g}$ of larval medium. This high larval density ensures that larvae experience stress and competition. In preliminary experiments these conditions resulted in $\sim 70 \%$ larval mortality.

At emergence, each fly was frozen at $-20^{\circ} \mathrm{C}$. Flies from the pure cups were dried for $72 \mathrm{~h}$ at $60^{\circ} \mathrm{C}$ and weighed to the nearest $0.1 \mathrm{mg}$. Both wings of all flies were mounted in Euparol, between two slides clamped with elastic bands to flatten the wings. Flies from mixed cups were electrophoresed to determine their parentage.

\section{Wing measurement/dry mass regression}

Four landmarks (the branching point of veins $R_{1}$ and $R_{3}$, the intersection of veins $C$ and $R_{1}$, the intersection of veins $\mathrm{C}$ and $\mathrm{M}_{1+2}$, and the intersection of the posterior crossvein and vein $\mathrm{M}_{3+4}$ ) were digitized in $x, y$ coordinates for the left wing of each fly using a dissecting microscope and camera lucida with a Summasketch III data tablet (Summagraphics, Seymour, Connecticut). The wing centroid size (square root of the summed squared distance of all landmark coordinates from the mean $x$ and $y$ coordinates of the landmarks) was calculated and regressed against the teneral dry mass for flies from the pure cups. No significant effects of 
experiment, sex, line and maternal age on the slope of this regression were found. Accordingly a single regression, based on all available data, was used to estimate the teneral mass of the flies from the mixed cups.

\section{Data analyses}

In the experimental design presented above, two fundamentally different types of competition were studied. In 'pure cups', offspring from young or old females from only one line of flies were in competition with each other. The data obtained permit comparisons of fitness/ biological parameters of offspring of young and old females from three different lines, but the data for each group of flies were derived from individuals that were competing only against others of the same strain and cohort. In mixed cups, larvae were competing against members of their own line and cohort at the same time that they were competing against members of a different line; the mothers of the larvae from the competing lines may have been the same or different ages (Fig. 1). The data obtained in mixed cup experiments provide fitness/ biological data for two populations (that differed genetically and in some cases that differed also in the age of their mothers) that were in direct competition with each other, as well as their own kind. Outcomes of these two types of competition will differ if distinct kinds of larvae differ in parameters that affect larval performance under competition.

We chose to keep the analysis of pure and mixed cups entirely separate, because they are essentially different experiments. It is possible to partially combine the results into unbalanced analysis of variance (ANOVA) models (e.g. Novak et al., 1993), but it is not a completely satisfactory method and introduces several problems. Because the experimental unit is the replicate cup, and not each fly produced, we calculated a single value from the flies emerging from each cup for sex ratio, viability, teneral mass, and development time. To examine sex ratio we calculated the angular transformed proportion of male emergents for each replicate cup.

Viability in pure cultures was analysed using angular transformed survival. Viability in mixed cups was analysed using natural log-transformed Haldane's $V=(a /(b+1)) /(A / B)$, where $a$ and $b$ are the number of adults emerging and $A$ and $B$ are the number of larvae seeded into the culture from each line (Haldane, 1956; Santos et al., 1992). In order to reduce the number of cups for which we were unable to calculate $V$, because of missing values, we chose the line with the fewest number of cups with zero emergence for the numerators in the calculation of $V$.

For pure cups the use of mean mass was inappropriate because the same number of larvae were not competing throughout the experiment in each cup, given differences in larval mortality, and we were unable to use the number of emergents to correct for differences in effective larval density, since the assumptions of analysis of covariance were not met. We used natural log-transformed total biomass in ANOVAs of line by age. Thus, we were assessing effects on productivity and not on fly size per se. For mixed cups we used the ratio of the mean mass of each type of fly. Ratios were calculated with the mean of the more successful line, in terms of viability, as the numerator. Mean development time was analysed in pure cups because development time was not correlated with survival in these experiments. For mixed cups we used the ratio of the mean development time of each type of fly, calculated in the same fashion as the ratio of mean mass.

For each experiment we tested all variables in separate two-way (line by maternal age) and three-way (parental age line A by parental age line B by seeding ratio) ANOvAs for pure cups and mixed cups, respectively. There were several mixed cups in the study that produced emergents from only one line. This forced an unbalanced analysis because of missing values.

\section{Results}

\section{Parental differences}

With the exception of a single contrast (experiment 1, line D) there were no significant differences between the estimated masses of young and old females, but eggs from old females were significantly smaller than those from young females in four out of seven cases after Bonferroni correction (Table 1). Eggs of old females had significantly lower viability than those of young females in two of seven comparisons, but the difference was in the same direction in all cases (Table 1). After pooling data, egg hatch differed significantly between maternal ages (Young: $88.3 \%$; Old: $76.6 \%$; $\left(\chi^{2}=16.58, P<0.001\right.$, d.f. $\left.=1\right)$, lines (Line C: 93.0\%; Line D: 76.7\%; Line G: 80.5\%; $\left(\chi^{2}=22.82, \quad P<0.001, \quad\right.$ d.f. $\left.=2\right), \quad$ and experiments $\left(\operatorname{Exp} 1: 88.0 \% ; \operatorname{Exp} 2: 80.5 \% ; \operatorname{Exp} 3: 80.0 \% ;\left(\chi^{2}=\right.\right.$ $6.021, P=0.049$, d.f. $=2)$. In a randomized block ANOvA with line and maternal age as factors and with experiments as blocks, mothers for experiment 1 were slightly smaller than were mothers for the other experiments $\left(F_{2,216}=4.17, P=0.016, R^{2}=0.036\right)$, but produced slightly larger eggs $\left(F_{2,342}=8.82, P=0.0002, R^{2}=\right.$ $0.047)$. There were no line, age, or interaction effects for mass or egg volume.

\section{Overall survival and sex ratio}

Total survival in experiment $3(\mathrm{~m}+\mathrm{p})$ was $12.7 \%$, much lower than the survival rates of 26.0 and $25.3 \%$ 
Table 1 Mean $( \pm$ SE) maternal teneral mass, egg size, and hatch rate for young and old female hoyuse flies from lines used in three competition experiments. $P$-values are from two-tailed $t$-tests for maternal and egg size, before Bonferroni correction. For hatch rate $P$-values are from $\chi^{2}$-tests, before Bonferroni correction. Sample sizes for each group are 16, 25, and 50 for estimates of female mass, egg volume, and hatch rate, respectively

\begin{tabular}{|c|c|c|c|c|c|c|c|c|c|c|}
\hline \multirow[b]{2}{*}{ Experiment } & \multirow[b]{2}{*}{ Line } & \multicolumn{3}{|c|}{ Female mass (mg) } & \multicolumn{3}{|c|}{ Egg volume $\left(\mathrm{mm}^{3} \times 10^{3}\right)$} & \multicolumn{3}{|c|}{ Hatch rate $(\%)$} \\
\hline & & Young & Old & $P$ & Young & Old & $P$ & Young & Old & $P$ \\
\hline 1 & $\mathrm{C}$ & $4.4 \pm 0.12$ & $4.0 \pm 0.12$ & 0.031 & $56.2 \pm 0.88$ & $51.5 \pm 1.12$ & $0.002 *$ & 96 & 94 & 0.646 \\
\hline 1 & $\mathrm{D}$ & $3.3 \pm 0.12$ & $4.0 \pm 0.10$ & $0.001 *$ & $55.9 \pm 1.12$ & $51.2 \pm 1.34$ & $0.001 *$ & 76 & 86 & 0.202 \\
\hline 2 & $\mathrm{D}$ & $4.2 \pm 0.11$ & $4.0 \pm 0.11$ & 0.152 & $50.9 \pm 1.28$ & $53.0 \pm 1.22$ & 0.255 & 86 & 72 & 0.086 \\
\hline 2 & $\mathrm{G}$ & $4.0 \pm 0.12$ & $4.2 \pm 0.11$ & 0.297 & $51.8 \pm 1.14$ & $45.2 \pm 0.89$ & $0.001^{*}$ & 98 & 66 & $0.001 *$ \\
\hline 3 & $\mathrm{C}$ & $4.0 \pm 0.11$ & $3.9 \pm 0.10$ & 0.626 & $52.1 \pm 1.13$ & $48.9 \pm 1.13$ & 0.048 & 94 & 88 & 0.295 \\
\hline 3 & $\mathrm{D}$ & $4.6 \pm 0.17$ & $4.2 \pm 0.14$ & 0.086 & $47.2 \pm 1.07$ & $49.0 \pm 1.23$ & 0.260 & 78 & 62 & 0.081 \\
\hline 3 & G & $4.1 \pm 0.09$ & $4.2 \pm 0.08$ & 0.394 & $55.0 \pm 0.90$ & $49.6 \pm 1.48$ & $0.003^{*}$ & 90 & 68 & $0.007 *$ \\
\hline
\end{tabular}

${ }^{*} P<0.05$ after sequential Bonferroni correction.

from experiments $1(m+p)$ and $2(m+p)$, respectively. In experiment $3 \mathrm{~m}$ there were 10 cups that produced no adult and seven cups that produced flies from only one of the parental lines. The net result was greater apparent variability within groups and uneven sample sizes within cells of the ANOVA.

Of the 1910 flies that emerged in these experiments, $51.5 \%$ were male and the sex ratio did not depart significantly from $0.5\left(\chi^{2}=1.913\right.$, d.f. $\left.=1, P=0.166\right)$. Within experiments and in the pooled data there were no significant effects of experiment, line, or maternal age on sex ratio (analyses not shown). Accordingly, sex was excluded from the analyses of viability, mass, or development time.

\section{Pure cups}

In pure cups, offspring of old mothers had higher viability and greater total biomass than did offspring of young mothers (Fig. 2). Line $\mathrm{C}$ had higher survival than line $D$ in experiment $1 p$, and lower survival than line $G$ in experiment $3 p$, but in experiment $2 p$ survival of lines $\mathrm{D}$ and $\mathrm{G}$ did not differ. The maternal age effect differed significantly between lines in expts $2 p$ and $3 p$ (Fig. 2). Line and line-by-maternal age interaction did not affect biomass except in experiment $1 \mathrm{p}$, in which early line $\mathrm{C}$ larvae out-produced early line D larvae. There was no effect of maternal age on development time in expts $1 p$ and $2 p$, but in experiment $3 p$ late offspring developed more rapidly than early offspring and this difference was much greater in line $\mathrm{C}$ than in line $\mathrm{G}$ (Fig. 2).

\section{Mixed cups}

Maternal age affected viability in five out of six cases in the three mixed cup experiments, but the direction of the effect differed between lines and experiments (Table 2,
Fig. 3). Figure 3 contains plots of the performance of each maternal age for each line, relative to the two maternal ages from the competitor line. These plots are modifications of standard replacement plots (De Wit, 1960) designed to facilitate comparison of competitive differences between maternal age classes. Late $\mathrm{C}$ was better than early $\mathrm{C}$ when in competition with late $\mathrm{D}$ but there was no difference when competing with early $\mathrm{D}$ (Fig. 3a). Against line $\mathrm{G}$, late $\mathrm{C}$ did better than early $\mathrm{C}$, especially against late G (Fig. 3e). Early D was more competitive than late $\mathrm{D}$ against both ages of line $\mathrm{C}$ in experiment $1 \mathrm{~m}$ (Fig. $3 \mathrm{~b}$ ), but in experiment $2 \mathrm{~m}$ late $\mathrm{D}$ was better than early $D$ against both age classes of $G$, with a nonsignificant trend for the difference to be greater at lower seeding ratios of G:D larvae (Fig. 3d). Early $\mathrm{G}$ was slightly, but significantly, better than late $\mathrm{G}$ against both age classes of D (Fig. 3C). In experiment $3 \mathrm{~m}$ early $\mathrm{G}$ were marginally better than old $\mathrm{G}$ in competition with both age classes of $\mathrm{C}$ and there was a greater deficit of $\mathrm{C}$ emergents when the proportion of $\mathrm{C}$ larvae was high (Fig. 3F).

There were few significant effects of maternal age on competitor mass and development time ratios (Fig. 4). The pattern of effects is consistent with the observation that development time is negatively correlated with mass in these experiments $(r=-0.25, n=1900, P<0.0001)$. Early line D offspring achieved larger sizes and completed development more rapidly than their line $\mathrm{C}$ competitors (especially against late line C), but late D offspring were smaller and developed more slowly than their line $\mathrm{C}$ competitors. In experiment $1 \mathrm{~m}$ there was a small effect of seeding ratio on mass ratio, with line D 9\% smaller than line $\mathrm{C}$ at the $1: 1$ seeding ratio, but $>6 \%$ larger at the other ratios $\left(F_{2,22}=3.72, P=0.041\right)$. In experiment $2 \mathrm{~m}$, even though there were no effects on mass ratios, young line $\mathrm{G}$ flies completed development more rapidly than late line $\mathrm{G}$ did, relative to their line $\mathrm{D}$ 

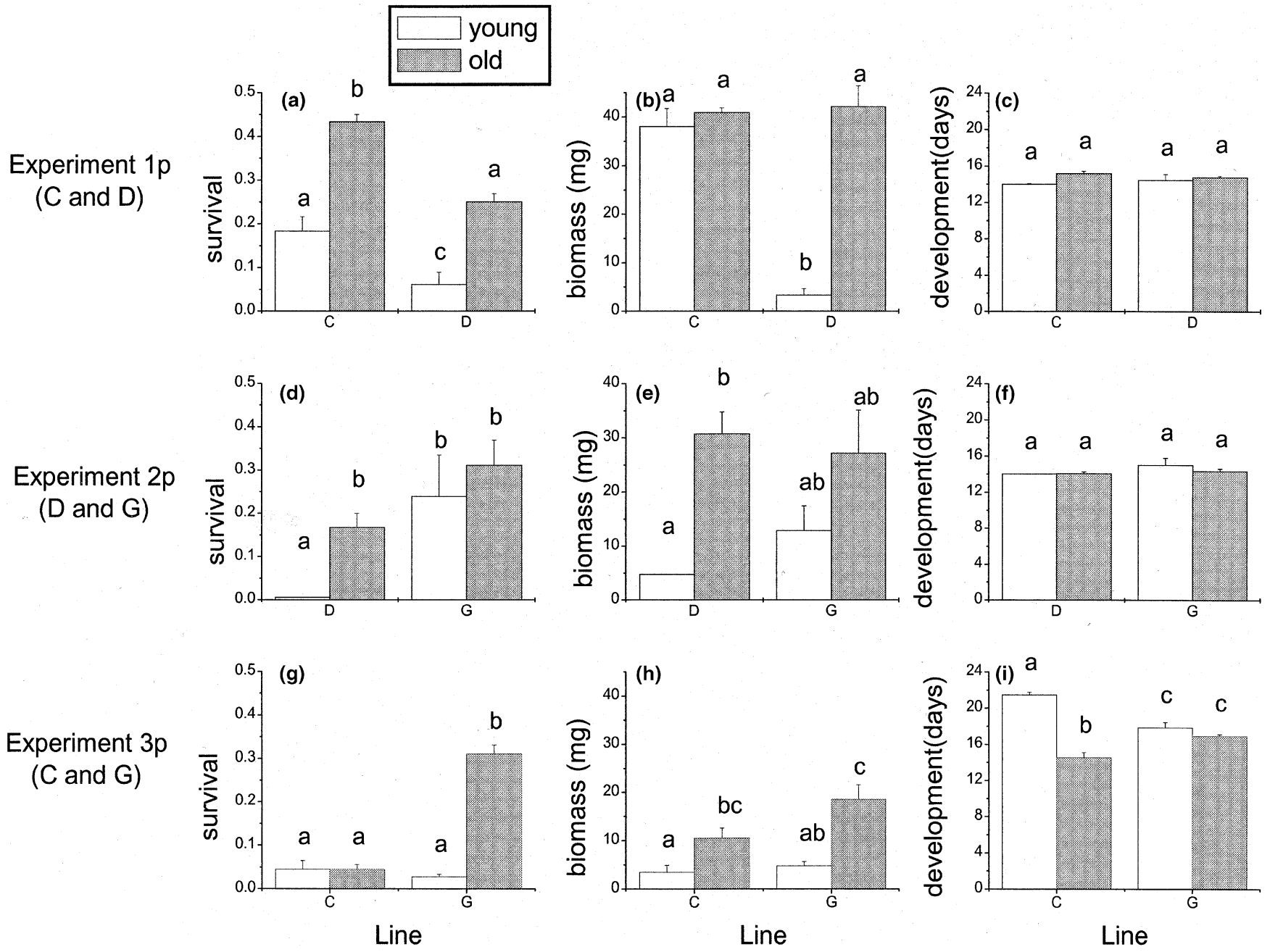

Fig. 2 Mean $( \pm \mathrm{SE})$ performance in pure culture of 60 larvae/3 $\mathrm{g}$ of larval medium from young and old mothers. For expt $1 \mathrm{p}$ : (a) proportion surviving per cup, (b) total biomass per cup, and (c) mean development time per cup. For expt 2p: (d) survival, (e) biomass, and (f) development time. For expt 3p: (g) survival, (h) biomass, and (i) development time. Lower case letters indicate significant differences between means using 2-way $T$-tests $(\alpha=0.05)$. Tests were made on angular transformed values for survival and on natural log transformed values for biomass and development time.

competitors $\left(F_{1,10}=13.99, P=0.004\right)$. There was also a significant interaction between seeding ratio and maternal age of line $G$ for development time. Early $G$ flies developed $24 \%$ more slowly than their D counterparts at the high G:D seeding ratio while late $\mathrm{G}$ flies took 3\% longer to develop than did their D counterparts at this ratio. At the other seeding ratios the development time ratios did not differ between maternal ages of line $G$ $\left(F_{2,10}=7.76, P=0.010\right)$. In experiment $3 \mathrm{~m}$ late line $\mathrm{G}$ flies were significantly larger relative to their line $\mathrm{C}$ competitors than were early $\mathrm{G}$ flies $\left(F_{1,10}=6.76\right.$, $P=0.027)$. There was also an interaction between seeding ratio and maternal age of line $\mathrm{G}$ for mass ratio. Late $\mathrm{G}$ flies were almost $40 \%$ heavier than line $\mathrm{C}$ flies when the G:C seeding ratio was $1: 3$, but at other ratios and maternal ages $\mathrm{G}$ flies were approximately $16 \%$ lighter than their $\mathrm{C}$ competitors $\left(F_{1,10}=6.58\right.$, $P=0.028)$.

\section{Discussion}

We have demonstrated that old female house flies can produce larvae that are more competitive, at high density, than larvae from early females. In most cases, maternal age was positively associated with larval viability, mass, and development rate. Competitive ability and the relationship of maternal age to competitive ability were strain specific. The strain- and mixture-specific nature of competitive ability has been demonstrated previously in house flies and constitutes strong evidence that larval competitive ability is under genetic control (Sokal \& Sullivan, 1963; Bhalla \& Sokal, 


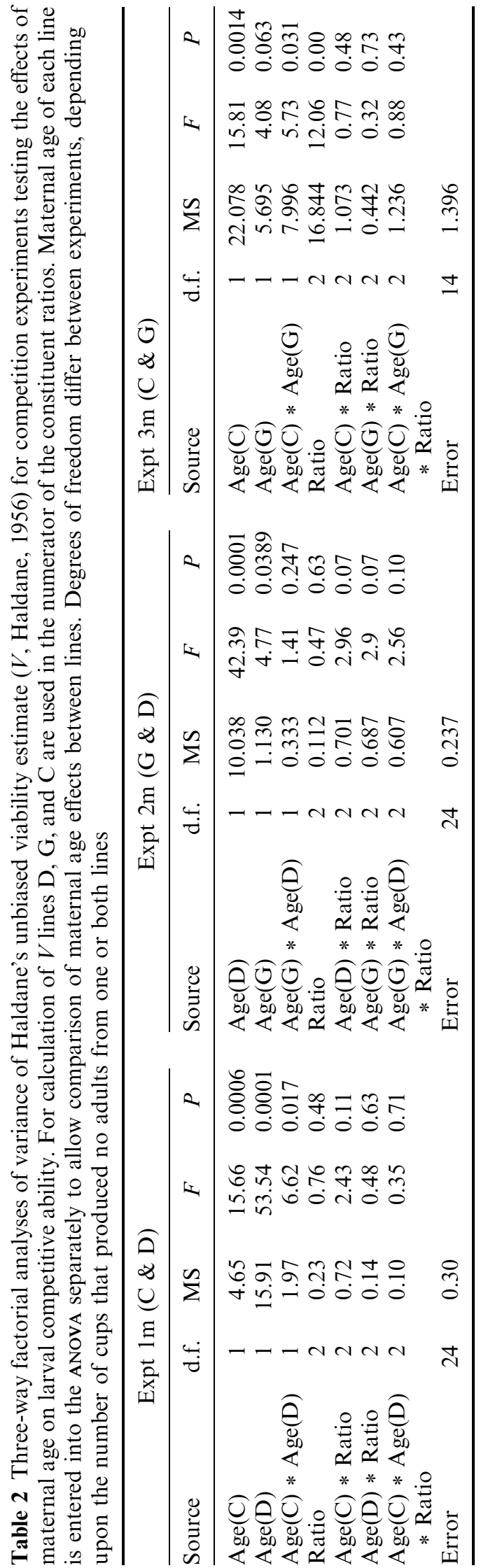

1964; Sullivan \& Sokal, 1965). Our results suggest that maternal age effects on larval competitive ability are also under genetic control.

Thus, maternal age effects in larval house flies are more than simple constraints of age-related physiological deterioration and they may be adaptive in nature. House fly populations increase throughout the summer in temperate areas (Black \& Krafsur, 1986). This implies that females will generally oviposit into more crowded situations as they age. Because larval density has strong life history consequences, females may experience evolutionary pressure to produce better competitors at advanced ages and the positive maternal age effects we observed on larval competitive ability may be a response to this pressure.

The basis of the competitive advantage of late larvae is unknown. Late larvae were less active than early larvae at the beginning of these experiments (qualitative observation) and they came from smaller eggs (Table 1). Egg size is not related to egg energy content in house flies (McIntyre \& Gooding, 2000) so late hatchlings may have disproportionately larger energy reserves. If initial nutrient levels are very low then more active larvae might do well at low density because they will condition the medium more rapidly (assuming that activity correlates with medium conditioning) and will search for and find sufficient food to keep them alive until bacterial growth accelerates. At high larval density, active larvae might exhaust their hatchling energy reserves and the initial food supply, and starve before medium conditioning takes effect. Less active larvae might be initially less prone to starvation in poor media and could outlast more active larvae. However, less active larvae might be at a disadvantage in nutrient-rich media and also less able to condition nutrient-poor media in the absence of more active larvae. This explanation fits our data for late D offspring, which performed poorly in pure culture but performed well when a few competitors, that performed well in pure culture, were present (Table 2, Fig. 3). Similarly, ge house fly mutants are poor nutrient conditioners that perform well in the presence of larvae with normal nutrient conditioning activity (Bhalla \& Sokal, 1964; Bryant, 1969).

If the $G r^{\mathrm{f}}$ or $O d h^{\mathrm{s}}$ allele (or closely linked genes) affect the rate of ageing in female house flies then this might explain line differences in maternal age effects. But line $\mathrm{G}$ (homozygous for $G r^{\mathrm{f}}$ ), which was least affected by maternal age in competition, exhibited the largest maternal age effects on egg size and viability (Table 1 ). Line D (homozygous for $O d h^{\mathrm{s}}$ ), exhibited less difference in egg hatch than other lines and egg size differences were also small, while D was the only line with early larvae to dramatically outperform old larvae in any experiment $(1 \mathrm{~m})$. In this line, less age-related change in

(C) The Genetical Society of Great Britain, Heredity, 85, 480-489. 
Fig. 3 Modified replacement plots (see text) of competition between larvae produced by house flies from two maternal age classes from three genetically selected lines (C, D, and G). Each plot shows the performance (survivorship) of early and late offspring from one line in competition with early and late offspring from another line. (a) performance of $\mathrm{C}$ offspring in expt $1 \mathrm{~m}$; (b) performance of $\mathrm{D}$ offspring in expt $1 \mathrm{~m}$; (c) performance of $\mathrm{G}$ offspring in expt $2 \mathrm{~m}$; (d) performance of $\mathrm{D}$ offspring in expt $2 \mathrm{~m}$; (e) performance of $\mathrm{C}$ offspring in expt $3 \mathrm{~m}$ (f) performance of $\mathrm{G}$ offspring in expt $3 \mathrm{~m}$. Squares and circles are the performance of late and early offspring, respectively. Solid and hollow symbols indicate competition against early and late competitor offspring, respectively. In the absence of maternal age and line effects the expected proportion of emergents is equal to $\mathrm{X}$ in plots $\mathrm{A}, \mathrm{C}$, and $\mathrm{E}$ and $(\mathrm{X}-1)$ in plots $\mathrm{B}, \mathrm{D}$, and $\mathrm{F}$, where $\mathrm{X}$ is the $x$-axis value.
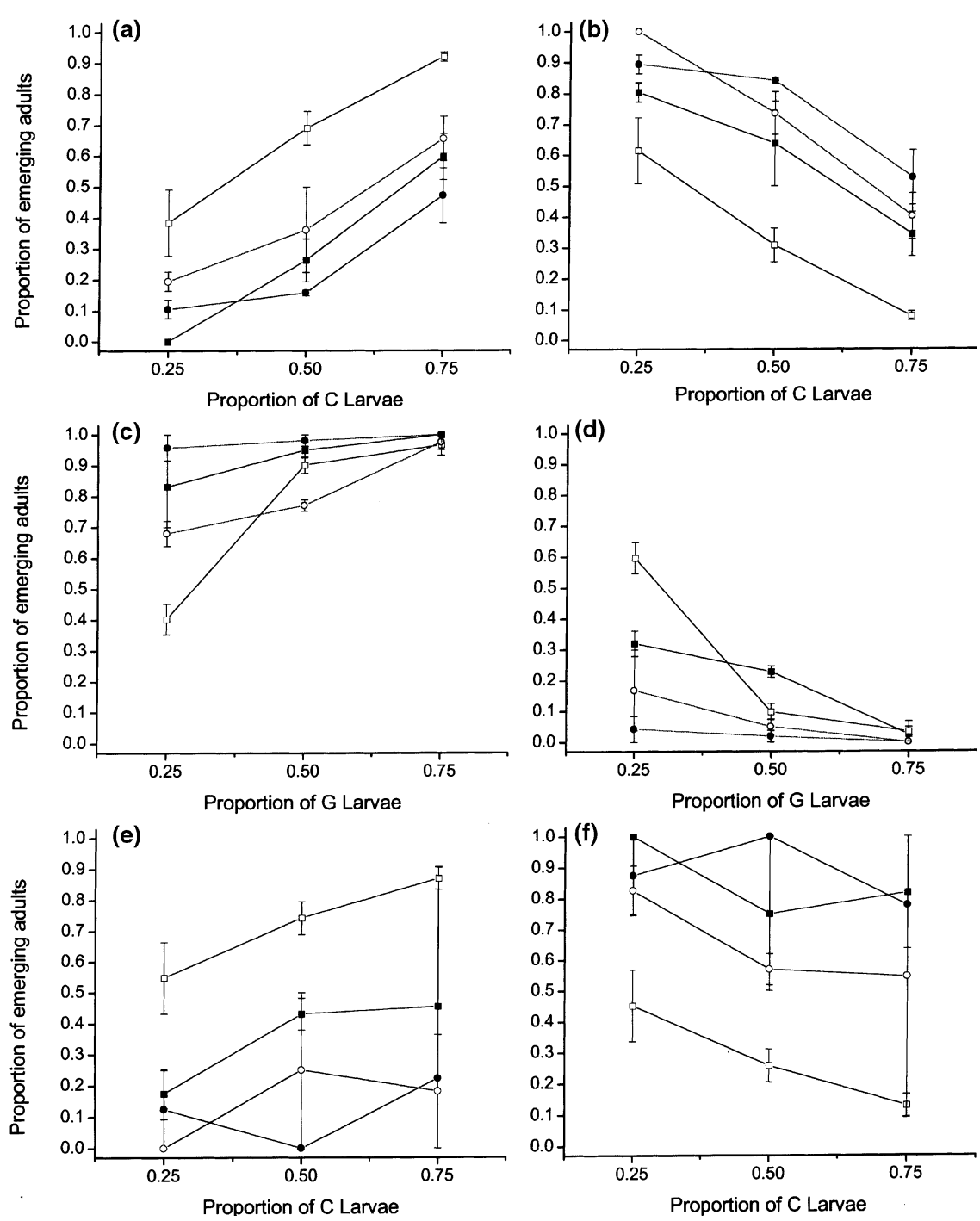

egg parameters may be related to less extreme maternal age effects upon larvae, but this does not explain the very weak performance of offspring of young D mothers in pure culture (expts $1 p$ and $2 p$ ).

One of the most salient features of this study is that the competitive effects of line, maternal age, and competition ratio could not be generalized. Rather competitive ability varied among combinations of lines and also between pure cups and mixed cups as has been demonstrated previously in house flies and Drosophila spp. (Lewontin, 1955; Lewontin \& Matsuo, 1963; Sokal \& Sullivan, 1963; Bhalla \& Sokal, 1964; Sullivan \& Sokal, 1965). The difficulty in predicting performance in mixtures from performance in pure culture has led to the use of tester strains to indirectly determine relative competitive abilities of genotypes (e.g. Santos et al., 1992). But, as argued in the introduction, this requires that either there is a single major determinant of competitive success or the different genotypes interact with each other in a common fashion. These assumptions are usually not demonstrated prior to use of the tester method. In Drosophila, for which the tester method is frequently used (e.g. Mueller, 1988; Santos et al., 1992; James \& Partridge, 1998), feeding rate is often highly correlated with larval success and is sometimes taken as the primary determinant of larval success (Bakker, 1961; Mueller, 1988). However, D. melanogaster strains are differentially susceptible to metabolic waste accumulation (Weisbrot, 1966; Botella et al., 1985), and subpopulations exhibit distinct, genetically based strategies for the utilization of the larval medium before and after larval density and waste concentrations increase (Borash et al., 1998). In larval competition studies using Drosophila, yeast growth has been viewed as an undesirable complication and many larval culturing methods provide killed yeast as food in sterile media (Bakker, 1961; Nunney, 1983), in spite of early demonstrations that live yeast interact with 


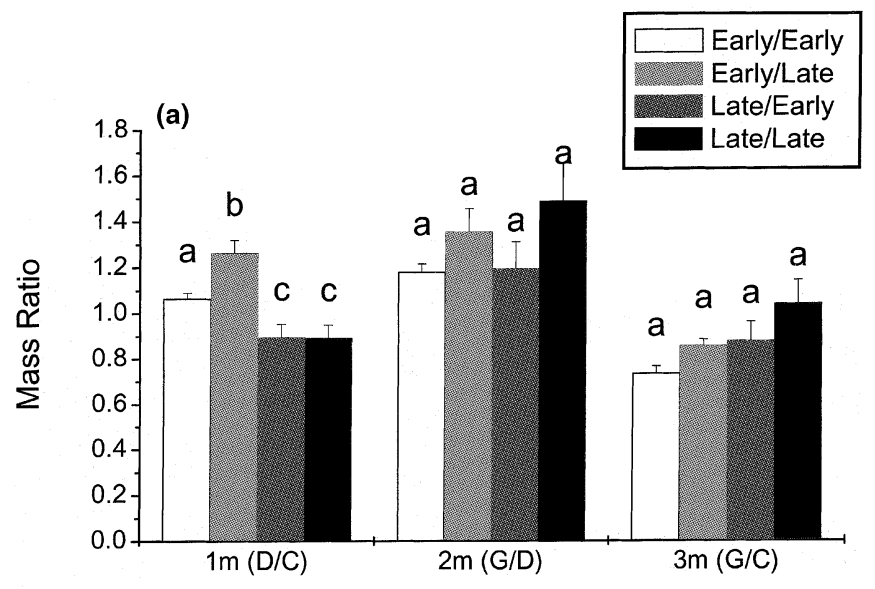

(b)

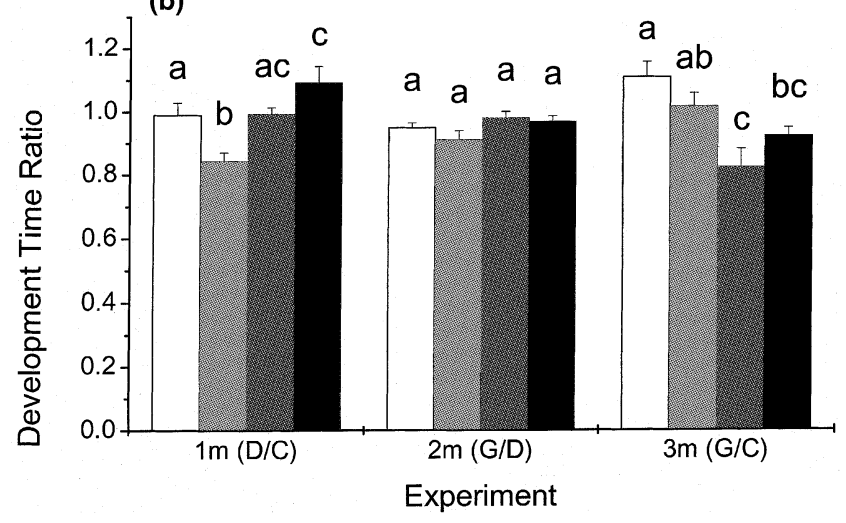

Fig. 4 Mean ( \pm SE) of ratios of mean fly mass (a) and mean development time (b) for flies from mixed cups in each of three competition experiments. In each experiment the ratio is the mean for the line indicated by the first letter in the $x$-axis label over the mean for the line indicated by the second letter. The legend indicates the maternal age class comparisons within each experiment (e.g. the leftmost bar in each plot is the mean ratio of values for larvae from young $\mathrm{D}$ mothers over the values for larvae from young $\mathrm{C}$ mothers). Lower case letters indicate significant differences between untransformed means using 2-way $T$-tests $(\alpha=0.05)$. Note: if the mass ratio is $>1$ then the first member of the comparison was heavier than the second, but if the development time ratio is $>1$ then the first member developed less rapidly than the second did.

Drosophila larvae (e.g. Gordon \& Sang, 1941). This eliminates a facet of the hatchling/microbial interaction known to be important in species with similar larval ecology. Thus, in Drosophila there is more than a single factor important in determining larval competitive ability. It is therefore inadvisable to adopt the tester stock method unless the factors acting in a particular contrast are known to be identical to those in other comparisons of interest.

Would the tester strain approach have been useful here? In our competition experiments (mixed cups only) using larval survivorship as the measure of success and ignoring maternal age effects, there are three independent rankings of larval competitive ability: $\mathrm{D}>\mathrm{C}$, $\mathrm{G} \gg \mathrm{D}$, and $\mathrm{G}>\mathrm{C}$. If line $\mathrm{C}$ had been the tester stock we would have predicted that $\mathrm{G} \sim \mathrm{D}$, which was not the case in experiment $2 \mathrm{~m}$. If line $\mathrm{D}$ were the tester strain the resulting prediction is that $G \gg \mathrm{C}$, which is in the correct direction, but the observed magnitude of the difference is smaller than predicted. If line $\mathrm{G}$ were the tester strain, the prediction that $\mathrm{C}>\mathrm{D}$ is in the wrong direction. Thus we have a correct qualitative prediction in only a single case, but the magnitude is less than expected. The use of tester strain competition experiments is clearly suspect in house flies and probably also in other species with complex larval competition dynamics. We invite detractors to develop analytical models demonstrating that the tester methodology is sound in cases with complex larval dynamics. Existing models of larval competition consider only the amount of food available and the feeding rate (De Jong, 1976; Nunney, 1983), which reduces to a single larval trait (feeding rate).

\section{Acknowledgements}

This work improved by comments from two anonymous reviewers and was supported, in part, by a grant (A-3900) from the Natural Sciences and Engineering Research Council of Canada, to R.H.G.

\section{References}

BAKKER, K. 1961. An analysis of factors which determine success in competition for food among larvae of Drosophila melanogaster. Arch. Neerl. Zool., 14, 200-281.

BHAlla, S. C. AND SOKAL, R. R. 1964. Competition among genotypes in the housefly at varying densities and proportions (the green strain). Evolution, 18, 312-330.

BLACK IV, w. C. AND KRAFSUR, E. S. 1986. Population biology and genetics of winter house fly (Diptera: Muscidae) populations. Ann. Entomol. Soc. Am., 79, 636-644.

BORASH, D. J., GIBBS, A. G., JOSHI, A. AND MUELLER, L. D. 1998. A genetic polymorphism maintained by natural selection in a temporally varying environment. Am. Nat., 151, 148-156.

Botella, L. M., MOYA, A., GONZALEZ, M. C. AND MENSUA, J. L. 1985. Larval stop, delayed development and survival in overcrowded cultures of Drosophila melanogaster: effect of urea and uric acid. J. Insect Physiol., 31, 179-185.

BRYANT, E. H. 1969. The fates of immatures in mixtures of two housefly strains. Ecology, 50, 1049-4069.

BRYANT, E. H. AND SOKAL, R. R. 1967. The fate of immature housefly populations at low and high densities. Res. Popul. Ecol., 9, 19-44.

BRYANT, E. H. AND SOKAL, R. R. 1968. Genetic differences affecting the fates of immature housefly populations at two densities. Res. Popul. Ecol., 10, 140-170. 
DE JONG, G. 1976. A model of competition for food. I. Frequency dependent viabilities. Am. Nat., 110, 1013-1027. DE WIT, C. R. 1960. On competition. Versl. Landb. Ond., 66, $1-82$.

DENLINGER, D. L. 1998. Maternal control of fly diapause. In: Mousseau, T. A. and Fox, C. W. (eds) Maternal Effects as Adaptations, pp. 275-287. Oxford University Press, New York.

GORDON, C. AND SANG, J. H. 1941. The relation between nutrition and exhibition of the gene Antennaless (Drosophila melanogaster). Proc. R. Soc. B, 130, 151-184.

HALDANE, J. B. S. 1956. Estimation of viabilities. J. Genet., 54, 294-296.

JAMES, A. C. AND PARTRIDGE, L. 1998. Geographic variation in competitive ability in Drosophila melanogaster. Am. Nat., 151, 530-537.

KENCE, M. AND JDEIDI, T. 1997. Effect of malathion on larval competition in house fly (Diptera: Muscidae) populations. J. Econ. Entomol., 90, 59-65.

LEWONTIN, R. C. 1955. The effects of population density and composition on viability in Drosophila melanogaster. Evolution, 9, 27-41.

LEWONTIN, R. C. AND MATSUO, Y. 1963. Interaction of genotypes detemining viability in Drosophila busckii. Proc. Natl. Acad. Sci. U.S.A., 49, 270-278.

MATHER, K. AND CAligaRI, P. D. S. 1981. Competitive interactions in Drosophila melanogaster. II. Measurement of competition. Heredity, 46, 239-254.

McINTYRE, G. S. 2000. Maternal effects on house fly offspring quality. $\mathrm{PhD}$ thesis, University of Alberta.

McINTYRe, G. S. AND GOODING, R. H. 1995. Pteridine accumulation in Musca domestica. J. Insect Physiol., 41, 357-368.

McINTYRE, G. S. AND GOODING, R. H. 1998. Effect of maternal age on offspring quality in tsetse (Diptera: Glossinidae). J. Med. Ent., 35, 210-215.

McINTYRE, G. S. AND GOODING, R. H. 2000. Egg size, contents, and quality: maternal-age and -size effects on house fly eggs. Can. J. Zool., 78, 1544-1551.

MOUSSEAU, T. A. AND DINGLE, H. 1991a. Maternal effects in insect life histories. Ann. Rev. Entomol., 36, 511-534.

MOUSSEAU, T. A. AND DINGLE, H. 1991b. Maternal effects in insects: examples, constraints, and geographic variation. In: Dudley, E. C. (ed.) The Unity of Evolutionary Biology, Vol. II, pp. 745-761. Dioscoridies Press, Portland, OR.
MousseAu, T. A. AND FOX, C. W. 1998. Maternal Effects as Adaptations. Oxford University Press, New York.

MUELLER, L. D. 1988. Evolution of competitive ability in Drosophila by density-dependent natural selection. Proc. Natl. Acad. Sci. U.S.A., 85, 4383-4386.

MURPHY, D. D., LAUNER, A. E. AND EHRLICH, P. R. 1983. The role of adult feeding in egg production and population dynamics of the checkerspot butterfly Euphydryas editha. Oecologia, 56, 257-263.

NOVAK, M. G., HIGLEY, L. G., CHRISTIANSSEN, C. A. AND ROWLEY, w. A. 1993. Evaluating larval competition between Aedes albopictus and A. triseriatus (Diptera: culicidae) through replacement series experiments. Environ. Entomol., 22, 311-318.

NUNNEY, L. 1983. Sex differences in larval competition in Drosophila melanogaster: The testing of a competition model and its relevance to frequency-dependent selection. Am. Nat., 121, 67-93.

ROSSITER, M. C. 1991. Maternal effects generate variation in life history: consequences of egg weight plasticity in the gypsy moth. Funct. Ecol., 5, 386-393.

SANTOS, M., FOWLER, K. AND PARTRIDGE, L. 1992. On the use of tester stocks to predict the competitive ability of genotypes. Heredity, 69, 489-495.

SCHMIDTMANN, E. T. AND MARTIN, P. A. W. 1992. Relationship between selected bacteria and the growth of immature house flies, Musca domestica, in an axenic test system. J. Med. Ent., 29, 232-235.

SOKAL, R. R. AND Sullivan, R. L. 1963. Competition between mutant and wild-type house-fly strains at varying densities. Ecology, 44, 314-322.

SUllivAN, R. L. AND SOKAL, R. R. 1963. The effects of larval density on several strains of the house fly. Ecology, 44, 120-130.

SULLIVAN, R. L. AND SOKAL, R. R. 1965. Further experiments on competition between strains of house flies. Ecology, 46, 172-182.

WEISBROT, D. R. 1966. Genotypic interactions among competing strains and species of Drosophila. Genetics, 53, 427-435. 